\title{
Reservado a evolución; muerte súbita abortada, reporte de un
} caso

\author{
Reserved for evolution; sudden death aborted, a case report \\ Gustavo Ruíz-González ${ }^{1 *}$, Marcos Alarcón-Michel${ }^{2}$ y Luis D. Sánchez-Mayo ${ }^{3}$ \\ ${ }^{1}$ Servicio de Emergencias Adultos; ${ }^{2}$ Departamento de Electrofisiología; ${ }^{3}$ Unidad de Cuidados Intensivos Cardiovasculares. Hospital Central Militar, \\ Ciudad de México, México
}

\section{Introducción}

Se considera muerte súbita (MS) la que ocurre de manera inesperada dentro de la primera hora desde el inicio de los síntomas, o si se produce en ausencia de testigos cuando el fallecido ha sido visto en buenas condiciones en menos de 24 horas antes de hallarlo muerto. Algunos pacientes fallecen instantáneamente, pero la mayoría tienen algunos pródromos ${ }^{1}$.

En el siglo XVIII se publicó el libro que por encargo del papa Clemente XI escribió Lancisi sobre los frecuentes casos de MS que ocurrían en Roma. Los estudios clínicos y necrópsicos pusieron de manifiesto la relación de la MS con la presencia de dolor precordial y signos anatomopatológicos de enfermedad coronaria $^{1,2}$. La MS probablemente sea el desafío más importante de la cardiología moderna, por el gran número de casos que existen (tan solo en los Estados Unidos de América ocurren más de 3000.000 al año) ${ }^{1,3}$.

La MS puede aparecer en el contexto de un síndrome coronario agudo $(\mathrm{SCA})^{2,3}$. Este síndrome abarca desde paro cardiaco, inestabilidad eléctrica o hemodinámica con shock cardiogénico debido a isquemia en curso, hasta pacientes que ya están libres de dolor nuevamente en el momento de la presentación ${ }^{1-3}$. El síntoma principal que inicia el diagnóstico y la cascada terapéutica en los pacientes con sospecha de SCA es el malestar torácico agudo descrito como dolor, presión, opresión y ardor ${ }^{1,2}$.

\section{Presentación del caso}

Varón en la octava década de vida con diagnóstico de SCA sin elevación del segmento ST que acude al servicio de urgencias a las 21:00 horas, trasladado desde el Hospital Militar Regional de Cuernavaca (México) tras deterioro de la clase funcional y síntomas anginosos atípicos de 24 horas de evolución, previamente tratado por un facultativo con vitaminas orales y analgésicos, sin mejoría. Tiene como antecedentes diabetes e hipertensión arterial de larga evolución con mal apego al tratamiento, así como "cirugía cardiaca" hace 10 años. Al ingreso los signos vitales son: presión arterial media $107 \mathrm{mmHg}$, frecuencia cardiaca 78 latidos por minuto, frecuencia respiratoria 20 respiraciones por minuto, saturación de oxígeno 95\% y temperatura $37.2{ }^{\circ} \mathrm{C}$. La exploración física muestra: sin alteración del estado de alerta, palidez de tegumentos ++/++++, adecuada mecánica ventilatoria sin soplos agregados, y resto sin datos de relevancia clínica. GRACE 144, TIMI Risk 3 (alto riesgo 13\%), Killip y Kimball 1.

\section{Abordaje}

Se realiza electrocardiograma de 12 derivaciones que muestra ritmo sinusal a 78 latidos por minuto, hipertrofia del ventrículo izquierdo (Sokolow 37) y ondas $Q$ en derivaciones inferiores (Fig. 1 A).

\section{Correspondencia:}

*Gustavo Ruíz-González

E-mail: cest.ruiztavo@gmail.com bajo la licencia CC BY-NC-ND (http://creativecommons.org/licenses/by-nc-nd/4.0/).
Fecha de recepción: 04-11-2021

Fecha de aceptación: 13-12-2021 DOI: 10.24875/REIE.21000138
Disponible en internet: 07-03-2022

Rev Educ Investig Emer. 2022;4(Supl 1):59-62 www.medicinadeemergencias.com México SA de CV. Este es un artículo open access 


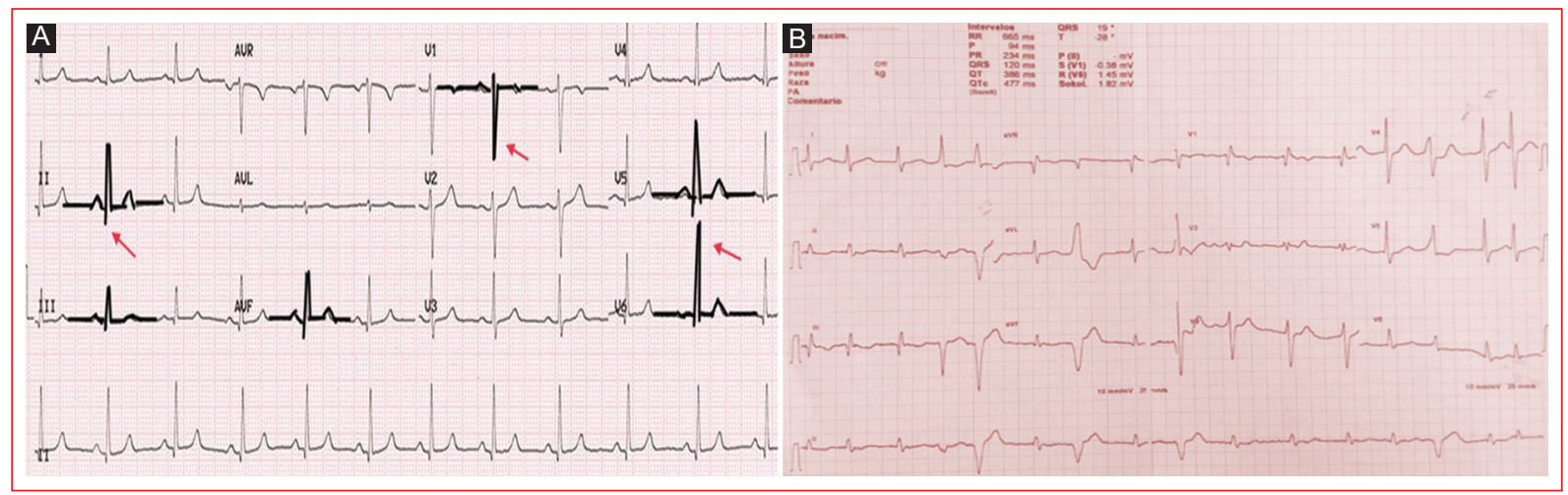

Figura 1. A: electrocardiograma de superficie, registrado al ingreso en la sala de emergencias, que muestra cambios sugestivos de daño miocárdico secundario a comorbilidad hipertensiva sistémica, patrón sugestivo de antecedente de necrosis de pared inferior (flechas). B: electrocardiograma en presencia de fibrilación auricular, bloqueo incompleto de rama derecha del haz de His, así como extrasístoles ventriculares posterior al retorno a la circulación espontánea.

Los laboratorios reportan: leucocitos 10.10, hemoglobina 10.80 , plaquetas 190 , linfocitos 1.40 , neutrófilos 8.40 , tiempo de protrombina 16.80 , tiempo de tromboplastina parcial 30, International Normalized Ratio 1.37, fibrinógeno 709, glucosa 261, urea 107, nitrógeno ureico en sangre 50 , creatinina 2.4 , sodio 132.5 , potasio 3.4 , calcio 7.52 , magnesio 2.1 , proteína $C$ reactiva 90 , creatina cinasa MB 2, mioglobina 176, péptido natriurético tipo B 1420 y dímero D 1350. Troponina I en algoritmo de 1 hora y 3 horas de $0.30 \mathrm{ng} / \mathrm{ml}$ para ambos. Prueba rápida de antígenos para COVID-19 negativa.

\section{Tratamiento}

Se inicia manejo con terapia antiplaquetaria dual (ácido acetilsalicílico dosis de carga $300 \mathrm{mg}$ y clopidrogel dosis carga $300 \mathrm{mg}$ ), heparina no fraccionada $70 \mathrm{U} / \mathrm{kg}$, atorvastatina $80 \mathrm{mg}$, apixabán $5 \mathrm{mg}$, furosemida $20 \mathrm{mg}$, espironolactona $25 \mathrm{mg}$, amiodarona $200 \mathrm{mg}$ y amlodipino $10 \mathrm{mg}$. Se decide su ingreso a piso con diagnóstico de SCA tipo infarto agudo al miocardio sin elevación del segmento ST, con plan de coronariografía diagnóstica dentro de las primeras 24 horas.

\section{Desenlace y seguimiento}

A las 07:00 horas presenta desaturación de oxígeno del $84 \%$ y síntomas anginosos atípicos. A las 07:05 horas se detecta taquicardia ventricular sin pulso, monomórfica sostenida. Ante el ritmo de paro, se realizan maniobras de reanimación avanzada administrando descarga con desfibrilador a $360 \mathrm{~J}$. Retorna a la circulación espontanea tras 10 minutos de maniobras, pasando a ritmo sinusal con paroxismos de fibrilación auricular (Fig. 1 B).

Pasa a sala de hemodinámica en la primera hora, se le realiza intervención coronaria percutánea, coronariografía diagnóstica y angioplastia, encontrando enfermedad multivaso con obstrucción total crónica en diferentes segmentos de las arterias descendente anterior, circunfleja (Fig. 2) y coronaria derecha desde su segmento ostial. Se observa un puente de la arteria mamaria izquierda a la arteria descendente anterior permeable y un puente venoso a la coronaria derecha permeable. Se implantan stents de la segunda obtusa marginal a la circunfleja, con angiografía final TIMI 3.

Tras el tratamiento de reperfusión coronaria, el paciente continuó presentando periodos cortos de taquicardia ventricular de 5-7 segundos. Para evitar hacer más episodios, al no contar en el área con chaleco desfibrilador, se le coloca un desfibrilador automático implantable (DAl) bicameral en modo DDD.

Los hallazgos ecocardioscópicos tras la reperfusión coronaria son: fracción de eyección del ventrículo izquierdo $34 \%$, ritmo de fibrilación auricular, dilatación global de cavidades cardiacas de predominio izquierdo/ hipertrofia concéntrica del ventrículo izquierdo con alteraciones del engrosamiento en reposo, acinesia de paredes inferior y posterolateral en sus tres segmentos, hipocinesia de pared lateral en sus tres segmentos, disfunción sistólica del ventrículo izquierdo/insuficiencia valvular mitral ligera de etiología funcional, función sistólica del ventrículo derecho limítrofe (desplazamiento sistólico del plano del anillo tricuspídeo $10 \mathrm{~mm}$ y fracción de acortamiento 36\%). 


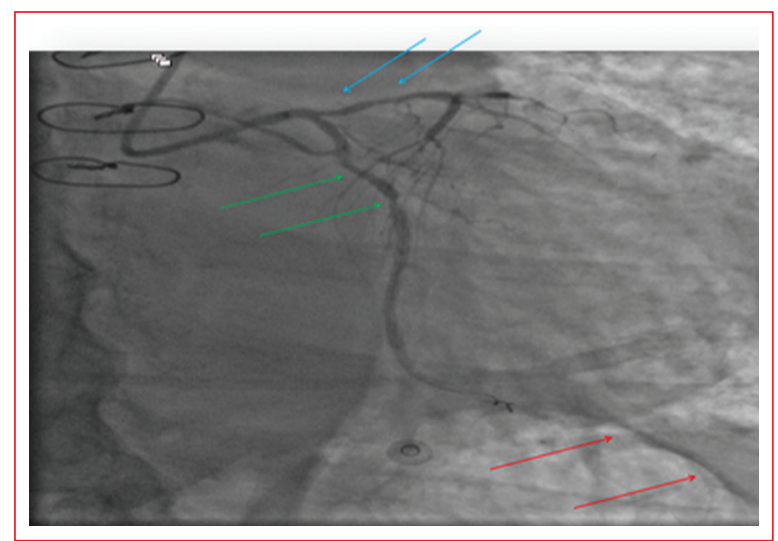

Figura 2. Coronariografía en la que se aprecian las siguientes lesiones: arteria descendente anterior con oclusión total crónica desde su segmento proximalmedio, ramo diagonal, y oclusión total crónica desde su segmento ostial (flechas azules); y arteria circunfleja con lesión crítica del $90 \%$ en el segundo ramo obtuso (flechas verdes) y obstrucción total crónica, moderadamente calcificada que se extiende del segmento medio hasta distal (flechas rojas).

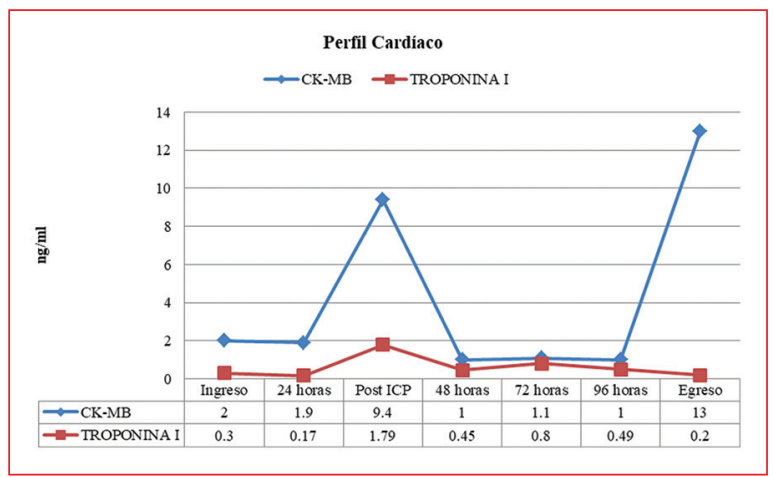

Figura 3. Comparación entre marcadores de daño miocárdico, presentando elevación de inicio y persistencia de la creatina cinasa (CK) MB mayor que de la troponina I desde el día de ingreso.

\section{Discusión}

En el abordaje inicial del paciente con datos anginosos, independientemente de su estado hemodinámico, la probabilidad de presentar eventos de MS cardiaca secundaria a la aparición de arritmia mortal (taquicardia ventricular) es muy alta ${ }^{2,4}$. Además de la sospecha de un síndrome coronario en evolución, se deben considerar otras afecciones de origen cardiovascular dentro del diagnóstico diferencial, aquellas que son potencialmente mortales pero también tratables, como la disección aórtica, la embolia pulmonar y el neumotórax por tensión ${ }^{4-6}$. Debe realizarse urgentemente una ecocardiografía a todos los pacientes con inestabilidad hemodinámica de posible origen cardiovascular ${ }^{4,5}$

En México, el principal sustrato para desarrollar taquicardia ventricular es la cardiopatía isquémica, principalmente en pacientes adultos, pero sobre todo en los ancianos ${ }^{1,6}$. La reperfusión urgente es primordial, ya que la isquemia suele desencadenar estas arritmias. Puede ser necesaria la cardioversión eléctrica o la desfibrilación repetida. Si no se logra un control adecuado, se debe considerar la administración intravenosa de amiodarona. la presentación típica de la arritmia es la taquicardia ventricular inestable, polimórfica y relativamente rápida, que suele degenerar en fibrilación ventricular. La taquicardia ventricular que se mantiene después de la fase aguda y no se desencadena por isquemia recurrente implica mal pronóstico; en estos casos, debe evaluarse la conveniencia de implantar un DAI para la prevención secundaria de la MS cardiaca ${ }^{6}$.

\section{Conclusiones}

Podemos contribuir a mejorar el pronóstico de los pacientes que sufren eventos de parada cardiorrespiratoria, reconocer e iniciar su abordaje desde el primer contacto en el servicio de emergencias, clasificar y determinar a los candidatos que requieran valoración y terapéutica multidisciplinaria, como es en el contexto de los síndromes coronarios, y así disminuir los eventos de MS cardiaca.

\section{Financiamiento}

Los autores no recibieron patrocinio para llevar a cabo este artículo.

\section{Conflicto de intereses}

Los autores declaran no tener conflictos de intereses.

\section{Responsabilidades éticas}

Protección de personas y animales. Los autores declaran que para esta investigación no se han realizado experimentos en seres humanos ni en animales.

Confidencialidad de los datos. Los autores declaran que han seguido los protocolos de su centro de trabajo sobre la publicación de datos de pacientes. 
Derecho a la privacidad y consentimiento informado. Los autores han obtenido el consentimiento informado de los pacientes y/o sujetos referidos en el artículo. Este documento obra en poder del autor de correspondencia.

\section{Bibliografía}

1. Bayés A, Elosua R. Muerte súbita. Rev Esp Cardiol. 2012;65:1039-52.

2. Collet J, Thiele H, Barbato E, Barthélémy $O$, Bauersachs J, Bhatt D, et al. 2020 ESC Guidelines for the management of acute coronary syndromes in patients presenting without persistent ST-segment elevation. Eur Heart J. 2021;42:1289-367.
3. Oraii A, Bozorgi A, Tajdini M. Differences in the 2020 ESC versus 2019 ACC/AHA/HRS guidelines on atrial fibrillation. Eur Heart J. 2021; 42:1820-1.

4. Stepinska J, Lettino M, Ahrens I, Bueno H, Garcia-Castrillo L Khoury A et al. Diagnosis and risk stratification of chest pain patients in the emergency department: focus on acute coronary syndromes. A position paper of the Acute Cardiovascular Care Association. Eur Heart J Acute Cardiovasc Care. 2020:9:76-89.

5. Ghadri JR, Wittstein IS, Prasad A, Sharkey S, Dote K, Akashi YJ, et al. International expert consensus document on Takotsubo syndrome (part II): diagnostic workup, outcome, and management. Eur Heart J. 2018;39:2047-62.

6. Ibáñez $\mathrm{B}$, James $\mathrm{S}$, Agewall $\mathrm{S}$, Antunes MJ, Bucciarelli-Ducci $\mathrm{C}$, Bueno H, et al. Guía ESC 2017 sobre el tratamiento del infarto agudo de miocardio en pacientes con elevación del segmento ST. Rev Esp Cardiol. 2017;70:1082.e1-61. 